\title{
Synchronization and Channel Estimation in Experimental M-QAM OFDM Radio over Fiber Systems Using CAZAC Based Training Preamble
}

\author{
Hum Nath Parajuli ${ }^{1}$, Haymen Shams ${ }^{2}$ and Eszter Udvary ${ }^{1}$ \\ ${ }^{1}$ Department of Broadband Info Communication and Electromagnetic Theory \\ Budapest University of Technology and Economics \\ Budapest, Hungary \\ ${ }^{2}$ Department of Electronic and Electrical Engineering, University College London (UCL), \\ Torrington Place, WC1E 7JE, London, UK \\ \{hum.nath.parajuli, udvary\}@hvt.bme.hu
}

\begin{abstract}
In this paper, we propose a new technique for synchronization and channel estimation in M-QAM OFDM radio over fiber (RoF) system by using constant amplitude zero autocorrelation (CAZAC) sequence based training preamble. Delay and correlate method is used to identify the training sequence in the received signal vector and to correct the symbol timing offset. For an optimum demodulation of OFDM signal, optimum down sampling offset position has to be identified before applying symbol timing algorithm. To solve this issue, we present the iterative method of finding optimum down sampling offset position. We show that the training preamble used for synchronization can also be applied to estimate the channel response using averaging technique. Moreover, we used the least square estimation based channel estimation method using pilot subcarriers and compare the results with training preamble based estimation.
\end{abstract}

Keywords-Channel estimation; frequency offset; OFDM; pilot; radio over fiber; synchronization; training sequence

\section{INTRODUCTION}

The wireless bandwidths at conventional radio frequency (RF) bands $(0.7-2.6 \mathrm{GHz})$ are not sufficient to fulfill the higher capacity demands [1]. Higher frequency bands such as millimeter-wave (mm-wave) bands are considered as a solution to overcome the problem of frequency congestion in current wireless transmission systems [2]. Due to the existence of the license free frequency band of $7 \mathrm{GHz}$ from 57 to 64 $\mathrm{GHz}$, extensive research interests are shown on this band. The major challenge at this band is huge signal attenuation [1-3]. This requires deployment of the large number of small cells to cover the geographical area. Thus, the base station (BS) should be as simple as possible to reduce the overall cost of the system. At higher frequency regime with optical fiber transmission the chromatic dispersion (CD) affects the system performance significantly. The photonic technologies with mm-wave signal generation capability are considered as suitable technologies to solve the above mentioned issues [4]. Such systems can be considered as a conventional radio over fiber (RoF) system with millimeter wave signal delivery capability.
In RoF systems with mm-wave generation, RF signal is generated and transmitted through low loss and huge bandwidth optical fiber. Such links have the ability to deliver RF signals into remote cells without deteriorating their characteristics (RF, modulation formats etc.). In such systems, all signal processing (e.g. signal generation, data modulation, up conversion etc.) can be provided in central station (CS) and, hence enables a simple, and low cost BS. This makes the deployment of large number of remote femto cells/ pico cells easier that can provide few Gbps wireless access and makes the network simple and scalable [3].

The target goal for $5 \mathrm{G}$ network is to provide 1-10 Gbps wireless access solution to the end user $[1,5]$. The current research trends at $60 \mathrm{GHz}$ range use direct detection as well as coherent detection technologies [6-8]. Due to the potential deployment of large number of small cells, complex BS transceiver is not cost effective. Therefore, simple modulation and detection methods are required. The multicarrier systems are essential to increase the spectral efficiency in future RoF systems. Since OFDM provide highly spectral efficient system and robust to the linear optical impairments such as chromatic dispersion(CD), it is still an attractive candidate for $5 \mathrm{G}$ research [9].The study of OFDM system also provides the basis for advanced multicarrier system such as FBMC (filter bank multicarrier) systems [10]. OFDM modulation is a simple and well studied method of multicarrier system which has also been studied in $60 \mathrm{GHz}$ radio over fiber systems with various design aspects [11-13].

One of the major problems of the OFDM system is to synchronize the transmitted and received signal. OFDM is very sensitive to the synchronization error which causes fast Fourier transform (FFT) window misalignment, hence, an imperfect demodulation. The synchronization problem can be considered as timing estimation as well as carrier frequency offset estimation (FOE). The timing estimation problem consists of correct down sampling and correct symbol alignment. The frequency offset causes misalignment of the inverse fast Fourier transform (IFFT) and FFT subcarriers resulting inter carrier interference (ICI). The frequency response of the channel is estimated through channel 
estimation method and it is used to equalize the channel. Several research works exists to solve these issues [14]. Training preamble based and pilot based methods are more common and widely applied in practical situations [14, 15]. Delay and correlate methods can be applied for symbol timing and frequency offset correction. In [16], Moose proposed a technique for FOE using repeated training preamble. This method was also employed by Schmidl for symbol timing estimation [17]. In a similar way, different repetitive preamble structures were designed to improve the accuracy by Minn [18], Park [19] and Ren [20]. In this paper, we propose an iterative method for finding the optimum position for down sampling offset and lowest errors. We use a training preamble based on Ren method and apply in experimental 16QAM OFDM RoF system. We show also the consequence of the inaccurate down sampling. Moreover, we use the same training preamble to estimate the channel response and to equalize the channel using averaging technique.

The organization of this paper is as follows. In section II, the descriptions of the implemented system model of optical transmission setup and signal processing methods are given. Section III presents brief theoretical background of used symbol time estimation; frequency offset estimation, and channel estimation methods. Section IV illustrates the proposed iterative method for finding the optimum down sampling offset position that improves the system performance. In section $\mathrm{V}$, the description of the least square (LS) channel estimation method with interpolation using pilot subcarriers and the training preamble based channel estimation using averaging technique are provided. Finally, section VI concludes the paper.

\section{SYSTEM DESCRIPTION}

Fig. 1 represents the simplified block diagram of the optical transmitter and receiver setups in the lab. In the optical transmitter, a distributed feedback (DFB) laser at $1553.7 \mathrm{~nm}$ wavelength with line width of $10 \mathrm{kHz}$ is used to generate an optical comb. The optical comb consists of a dual drive modulator and is driven with $15 \mathrm{GHz}$ electrical local oscillator (LO) signal generator. Then, the optical signal is filtered to generate two optical tones spaced by $54 \mathrm{GHz}$ by using wavelength selective switch (WSS). MATLAB routines are used to generate 16QAM OFDM signal which is electrically up converted to $6 \mathrm{GHz}$ and uploaded to Arbitrary Waveform Generator (AWG). Two output channels of the AWG are used to drive the optical IQ modulator with the same OFDM signal shifted in phase by $90^{\circ}$. The IQ modulator bias voltages are adjusted to generate single sideband (SSB) optical signal. Then, the modulated optical signal is amplified and filtered by optical band pass filter (OBPF) to remove the amplified spontaneous emission (ASE) noise before transmission over the fiber. In the optical receiver, the detected optical signal by the photodiode is amplified and mixed with another electrical $\mathrm{LO}$ at $54 \mathrm{GHz}$ to down-convert it to intermediate frequency (IF) at $6 \mathrm{GHz}$. Then, the signal is recorded by the real time scope and the data is processed offline using MATLAB code.
Fig. 2 shows the DSP block diagrams inside both the transmitter and receiver side. In the transmitter DSP; the OFDM signal is created using common OFDM coding techniques such as QAM mapping, serial to parallel conversion, IFFT and cyclic prefix (CP) insertion. The OFDM signal generation parameters are given in Table I. The pilot carriers are inserted to achieve channel estimation at the receiver and fixed preamble based training symbol is inserted for synchronization and used also for channel equalization. The root raised cosine (RRC) filter is used for pulse shaping with a roll off factor of 0.4 . Then, the OFDM signal is up converted to IF frequency at $6 \mathrm{GHz}$. After normalization, the signal is uploaded to the two AWG channels with same magnitude but with different phase. The AWG has a sampling rate of $50 \mathrm{GS} / \mathrm{s}$, which gives 10 samples/OFDM symbols points.
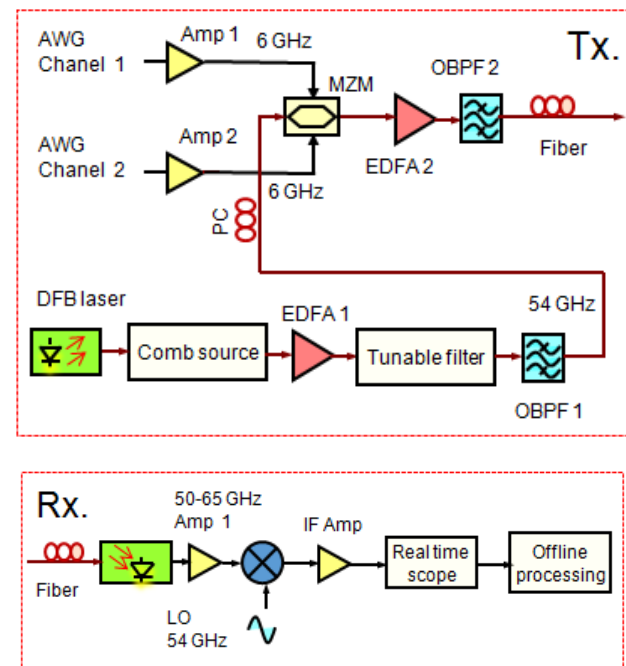

Fig.1. Experimental block diagram.

TABLE I. OFDM PARAMETERS
\begin{tabular}{|l|l|}
\hline \multicolumn{1}{|c|}{ parameter } & \multicolumn{1}{c|}{ value } \\
\hline No. of bits & 57344 \\
\hline Baud rate & 5 Gbaud \\
\hline QAM order & 16 \\
\hline CP & $25 \%$ \\
\hline NFFT & 1024 \\
\hline RRC roll off & 0.4 \\
\hline $\begin{array}{l}\text { Training } \\
\text { symbol }\end{array}$ & 1 \\
\hline Pilots & 5 \\
\hline
\end{tabular}

In the DSP at the receiver side, the received electrical signal from the scope is processed offline. The scope sampling rate is $80 \mathrm{GS} / \mathrm{s}$. Each symbol has 16 samples/symbol. The signal is first down converted to the baseband, and then filtered by a matched raised cosine filter with same roll off factor as in transmitter side. Afterword, all the signal processing algorithms are applied at the baseband signal. In order to obtain the subcarriers values, the baseband signal has to be down sampled into 1 sample/symbol. Therefore, the new 
proposed iterative method is used (described in section III) to pick the optimum down-sampling offset position. The imperfect selection of the sample point degrades the system performance. After down sampling, symbol timing alignment, and frequency offset compensation are performed. Then, the common OFDM decoding methods such as serial to parallel conversion, $\mathrm{CP}$ removal and FFT are processed in sequence as shown in the block diagrams in Fig. 2. The channel estimation is then applied using pilot subcarriers as well as training preamble for comparison. After removing pilots, parallel to serial conversion is done and QAM demodulation is performed.
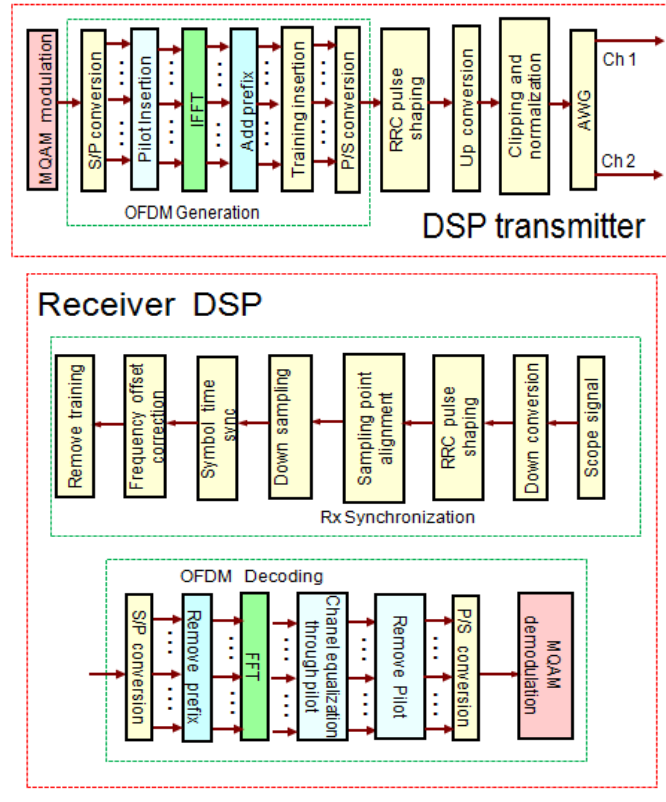

Fig.2. Simplified digital signal processing (DSP) block diagrams in transmitter side and receiver side.

Fig. 3 shows the structure of the OFDM symbols payload with training preamble symbol and pilot subcarriers. One training preamble symbol is used for synchronization and 5 pilot subcarriers are used for channel estimation. The pilots are inserted as a comb type at a same frequency interval.

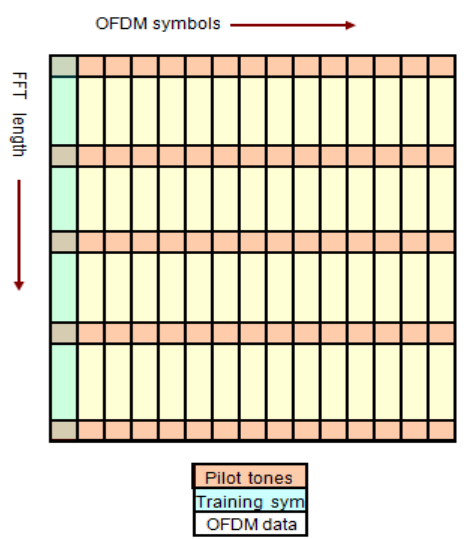

Fig.3. Structure of OFDM data payload with the training preamble symbol and pilot subcarriers. The pilot subcarriers are placed in comb type structure.

\section{THEORITICAL BACKGROUND OF SYNCHRONIZATION}

\section{A. Symbol timing estimation}

Symbol timing estimation in OFDM means finding the position in a received vector where the OFDM symbol starts. The error positioning of the sample points can be considered as a FFT window misalignment in transmission side and receiver side. To solve this problem, the starting sample position of IFFT and FFT should be aligned. The timing offset could be either in a leading or lag position from the best accurate position. For the leading offset, due to the use of cyclic prefix $(\mathrm{CP})$ the orthogonality between the subcarriers might still exist, and this introduces the phase rotation to all subcarriers. However, the lag offset will cause the misalignment in FFT window which will destroy the orthogonality among the subcarriers leading to ICI.

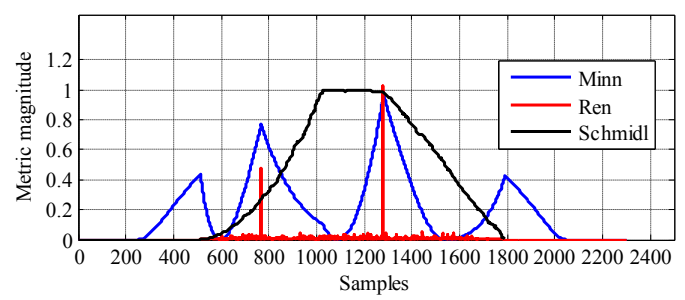

Fig.4. Timing metrics of different algorithms

To proceed with symbol timing algorithms, Schmidl [17], Minn [18] and Ren [20] methods are implemented in MATLAB and series of computer simulations were performed with AWGN noise for different SNR values. The illustration of timing metric of these methods is given in Fig. 4. In Schimdl method, the symbol starting position is at the end of flat region. The flat region arises from the existence of the CP. Due to this region; it is difficult to predict the accurate timing information for low SNR signals. For accurate estimation, it is required sharp slope between the flat region and sloppy region; in addition, the flat region should be strictly flat. In the Minn and Ren methods, the major peak provides the OFDM symbol starting position, and the minor peak is due to the used CP.

In our scheme of synchronization, we use the preamble sequence based on Ren's method. This method employs the constant envelop preamble based on constant amplitude zero auto-correlation (CAZAC) sequences. These sequences are weighted by the real valued pseudo noise (PN) sequence whose values are +1 or -1 . This preamble structure provides the constant peak to average power ratio (PAPR) and can be used as a training signal for all orders of modulations in $\mathrm{M}$ QAM OFDM. The preamble structure can be given as

$$
\text { Preamble }_{\text {Ren }}=\left[\begin{array}{ll}
(C A Z A C)_{\frac{N}{2}} & (C A Z A C)_{\frac{N}{2}}
\end{array}\right] \circ S_{N}
$$

where, $\circ$ is the Hardmard product operator which implies the element wise multiplication between the vectors, $N$ is the FFT 
length and $S$ is the real valued $\mathrm{PN}$ sequence. The timing metric can be calculated from equation (2), whose maximum peak gives the starting position of the OFDM symbol

Where,

$$
M_{\text {Ren }}(d)=\frac{\left|P_{\text {Ren }}(d)\right|^{2}}{\left(E_{\text {Ren }}(d)\right)^{2}} .
$$

$$
P_{\text {Ren }}(d)=\sum_{k=0}^{\frac{N}{2}-1} S_{k} \cdot S_{k+\frac{N}{2}} \cdot r^{*}(d+k) \cdot r\left(d+k+\frac{N}{2}\right)
$$

is the delay and correlate vector, $r$ is the received signal vector whose starting point has to be determined, $d$ is the time index with which delay and correlation task has to be accomplished. The energy vector $E_{\text {Ren }}(d)$ is used to normalize the correlation vector given by

$$
E_{\text {Ren }}(d)=\frac{1}{2} \sum_{k=0}^{\frac{N}{2}-1}|r(d+k)|^{2}
$$

After the timing error correction, the other signal processing tasks such as frequency offset correction and channel equalization can be achieved more appropriately.

\section{B. Frequency offset estimation (FOE)}

The frequency offset introduces the ICI caused by adjacent carriers. The frequency offset also causes the reduction of amplitude. The frequency offset can be calculated as [20]

$$
f_{\text {offset }}=\frac{1}{\pi} \text { angle }\left[P_{\text {Ren }}\left(t_{\text {start }}\right)\right]
$$

where, $t_{\text {start }}$ is the starting position of the OFDM symbol which is determined by equation (2). The frequency offset error is calculated by a fraction of the subcarrier spacing. Since the range of angle function is $\pm \pi$, equation (5) can estimate only \pm 1 range of subcarrier frequency spacing.

\section{PROPOSED METHOD OF SYMBOL TIMING ESTIMATION}

Due to DAC and ADC are not operating on the same sampling rate, it is required to find the accurate down sampling offset before applying the symbol timing algorithm. Even if DAC and ADC operate on the same sampling rate, optimum down sampling position needs to be determined. Due to the inaccurate sampling point the amplitude and phase of the extracted samples will not represent the valid symbols, and that degrades the system performance. To solve this issue, we construct an iterative algorithm as given in Fig. 5. With our method, the signal is first down sampled from $\mathrm{x}$ samples/symbol into 1 sample/symbol and then delay and auto correlation process is applied. The peak of autocorrelation gives the maximum amplitude for the sample which represents the correct starting symbol.

With the algorithm given in pseudo code of Fig. 5, the Ren metric given in equation (2) has to run for (ADC sampling rate)/(QAM symbol rate) times to calculate the peak values of each run. If the captured data from the scope is $\mathrm{x}$ times repeated transmitted signal, $\mathrm{x}$ numbers of peaks will appear in each iteration. Each peak represents the starting position of the OFDM symbol. For each iteration, the first peak is chosen and its value and position are stored in the buffer. Then, the maximum value of the peak is selected from the buffer. The position of the selected peak corresponds to the optimum down sampling offset position. After finding the optimum down sampling offset position, the signal is down-sampled at this optimum position to extract 1 sample/symbol. At this stage the FFT window will be at the optimum aligned position. After frequency offset correction OFDM decoding and channel estimation procedures can be applied.

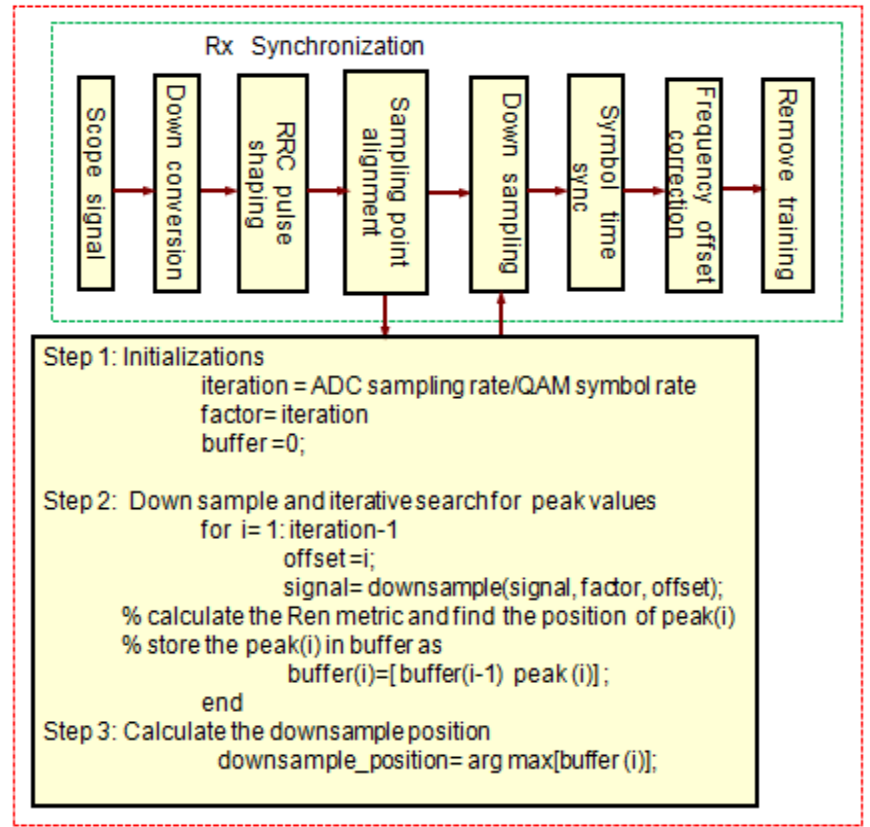

Fig. 5. Block diagram of optimum position for down sampling using iterative method.

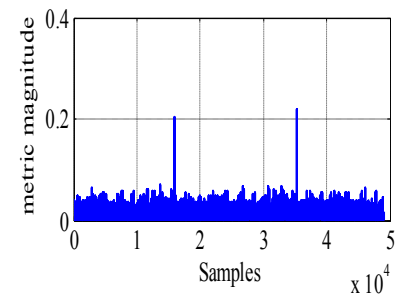

(a)

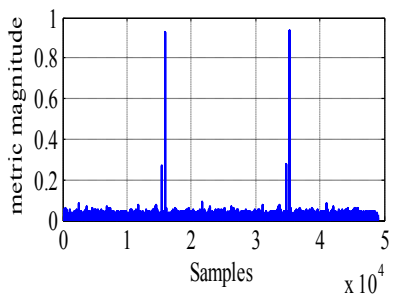

(b)
Fig. 6. Timing metric plots: (a) with incorrect down sampled signal (b) with optimum down sampled signal.

Fig. 6 shows the plots of timing metric of equation (2) for the cases of not correct offset position of down sampled signal, and the optimum down sampled signal. Two peaks in each figure results from the two times repetitions of the transmitted signal in the captured data from the scope. The lower peaks that are adjacent to the higher peak is due to the used CP. Due 
to the weak correlation, the peak values are lowered and noise level is increased in Fig. 6 (a). In Fig. 6 (b) the peak values are higher. These metric plots are for optical channel signal in which SNR is low. For higher SNR signals, the peak values appear with higher magnitude.

To observe the effect of inaccurate down sampling, Fig. 7 provides some illustrations. Fig. 7 (a) is the constellation diagram for the back-to-back configuration (without optical channel) with optimum offset position of the down sampled data. Fig. 7 (b) is the constellation diagram with not correct offset position. Fig. 7 (c), and Fig. 7 (d) are the constellation diagrams with optical channel with few meters of fiber with optimum, and not correct offset position of down sampled data, respectively. The constellations are affected by the amplitude dependent noise, could be due to the nonlinear distortion due to electrical amplifier and optical components. These experimental imperfections will be solved in future work.

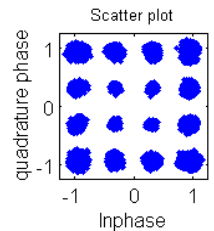

(a)

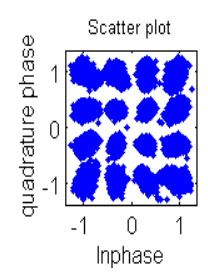

(c)

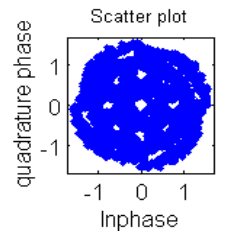

(b)

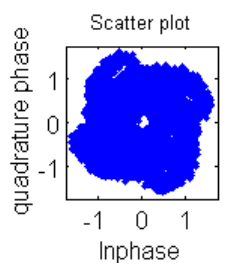

(d)
Fig. 7. . Constellation diagrams to illustrate the effect of optimum and not correct offset position of down sampling. (a) and (b) without optical channel (back to back) with optimum and not correct offset position of down sampled signals respectively. (c) and (d) with optical channel with few meters of fiber with optimum and not correct offset position of down sampled signal, respectively.

\section{Channel estimation Methods}

This process estimates the frequency response of the channel. Channel estimation is mandatory in the OFDM system to remove the channel degradation. Channel estimation can be done using training symbols or with pilot subcarriers along with interpolation.

\section{A. Least square (LS) estimation using pilot subcarriers}

Let us suppose $x(n)$ is the transmitted time domain symbols after IFFT and $y(n)$ is the received OFDM symbols in receiver after removing cyclic prefix. Where, $n=0,1,2 \ldots . . N-1, N$ is the FFT length. The FFT on $y(n)$ can be given as

$$
Y(k)=\frac{1}{N} \sum_{n=0}^{N-1} y(n) e^{-j 2 \pi \frac{k n}{N}}
$$

where, $k=0,1,2, \ldots, N-1$. With the channel response $H(k)$, transmitted signal response $X(k)$ and noise response $W(k)$, $Y(k)$ can be written as

$$
Y(k)=X(K) \cdot H(k)+W(k)
$$

The pilot symbols from $Y(k)$ and $X(k)$ are extracted and corresponding channel response can be estimated using

$$
\widehat{H}_{\text {pilot }}(k)=\frac{Y_{\text {pilot }}(k)}{X_{\text {pilot }}(k)} \text {. }
$$

To apply the channel estimation for equalizing OFDM signal, the interpolation method has to be used. After the interpolation, the size of the channel estimation vector $\widehat{H}_{\text {pilot }}(k)$ is the same size as in the vector $\widehat{H}(k)$. In our method, we apply the linear method of interpolation. In this type of interpolation, two pilots are used to estimate the channel response at the OFDM data subcarriers which are located between those pilots. In this way the pilots' channel response $\widehat{H}_{\text {pilot }}(k)$ is interpolated to $\widehat{H}(k)$.

\section{B. Training preamble based estimation}

We use the same training preamble structure for both synchronization and channel estimation. In our arrangement the training preamble symbol occupies all the subcarrier frequencies as given in Fig. 3. The frequency response of the channel can be calculated using LS method. To apply this channel response for equalizing the channel, we use the averaging method as given in Fig. 8 and compute the common channel response factors. The obtained response is applied to all OFDM data payload to equalize the channel.

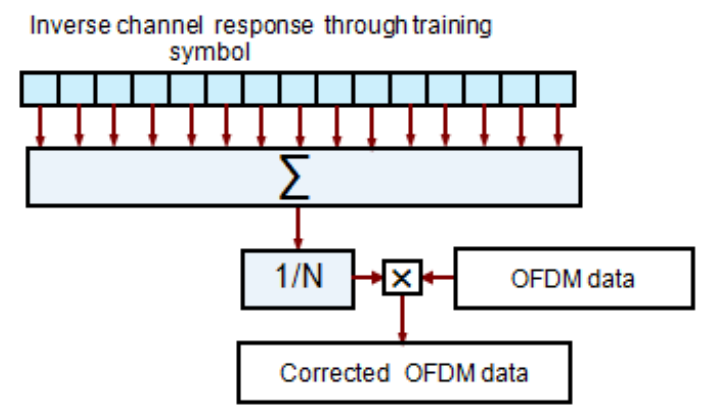

Fig. 8. Illustration of averaging method for correcting amplitude and phase response using training preamble.

Fig. 9 shows the comparison of the equalized constellation diagrams using the channel estimation of pilot subcarriers and training preamble. The channel estimation using training preamble along with averaging method has given improved constellation points than using pilot subcarriers. For better channel equalization the MMSE (minimum mean square error) method can be applied [21]. 


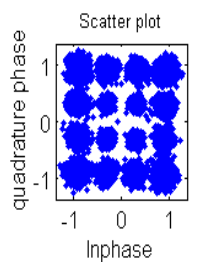

(a)

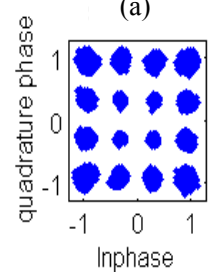

(c)

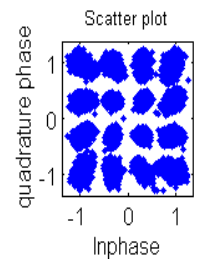

(b)

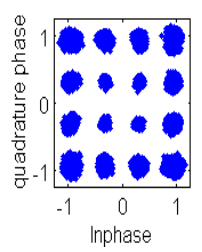

(d)
Fig. 9. Constellation diagrams of optical channel using few meter fiber length: (a) LS method with interpolation using pilots (b) averaging method using training preamble. Constellation diagrams of back to back (without optical channel): (c) LS method with interpolation using pilots (d) averaging method using training preamble.

\section{CONCLUSIONS}

In this paper, we proposed an iterative method for the synchronization and channel estimation for MQAM OFDM based radio over fiber (RoF) system based on constant amplitude zero auto-correlation (CAZAC) sequence preamble. We experimentally verified the use of this preamble for synchronization and channel estimation in 16QAM OFDM RoF by obtaining the optimum position of the down sampling. We also applied this preamble for equalizing the channel using averaging technique.

\section{ACKNOWLEDGMENT}

This work has been carried out within the project FiWiN5G, supported from European Union's Horizon 2020 research and innovation program.

\section{REFERENCES}

[1] T. S. Rappaport et al., "Millimeter Wave Mobile Communications for 5G Cellular: It Will Work!," in IEEE Access, vol. 1, pp. 335-349, 2013. doi: 10.1109/ACCESS.2013.2260813.

[2] L. Wei, R. Q. Hu, Y. Qian and G. Wu, "Key elements to enable millimeter wave communications for 5G wireless systems," in IEEE Wireless Communications, vol. 21, no. 6, pp. 136-143, December 2014. doi: 10.1109/MWC.2014.7000981.

[3] D. Novak et al., "Radio-Over-Fiber Technologies for Emerging Wireless Systems," in IEEE Journal of Quantum Electronics, vol. 52, no. 1, pp. 111, 2016. doi: 10.1109/JQE.2015.2504107.

[4] C. Lim et al., "Fiber-Wireless Networks and Subsystem Technologies," in Journal of Lightwave Technology, vol. 28, no. 4, pp. 390-405, Feb.15, 2010. doi: 10.1109/JLT.2009.2031423.

[5] G. K. Chang, L. Cheng, M. Xu and D. Guidotti, "Integrated fiber-wireless access architecture for mobile backhaul and fronthaul in 5G wireless data networks," 2014 IEEE Avionics, Fiber-Optics and Photonics Technology Conference (AVFOP), Atlanta, GA, 2014, pp. 49-50. doi: 10.1109/AVFOP.2014.6999461.

[6] C. T. Lin, W. E. Chen and H. T. Huang, "Simple 60-GHz Radio-overFiber System with Transmission Distance Improvement Employing
Signal-to-Signal Beat Noise Compensation," in IEEE Journal on Selected Areas in Communications, vol. 31, no. 12, pp. 773-779, December 2013. doi: 10.1109/JSAC.2013.SUP2.1213009

[7] A. Stöhr et al., "Highly spectral-efficient and high capacity millimeterwave wireless data transmission using a Coherent Radio-over-Fiber system (CRoF)," 2016 21st European Conference on Networks and Optical Communications (NOC), Lisbon, 2016, pp. 201-206. doi: 10.1109/NOC.2016.7507013.

[8] T. Shao, E. P. Martin, P. M. Anandarajah and L. P. Barry, "60-GHz Direct Modulation-Direct Detection OFDM-RoF System Using GainSwitched Laser," in IEEE Photonics Technology Letters, vol. 27, no. 2, pp.193-196, Jan.15, 2015. doi: 10.1109/LPT.2014.2364714.

[9] F. J. Effenberger, "Mobile backhaul and fronthaul systems," $201621 \mathrm{st}$ OptoElectronics and Communications Conference (OECC) held jointly with 2016 International Conference on Photonics in Switching (PS), Niigata, Japan, 2016, pp. 1-3.

[10] J. Zhang; M. Xu; J. Wang; F. Lu; L. Cheng; H. Cho; K. Ying; J. Yu; G. k. Chang, "Full-Duplex Quasi-Gapless Carrier-Aggregation Using FBMC in Centralized Radio-Over-Fiber Heterogeneous Networks," in Journal of Lightwave Technology, no.99, pp.1-1, $2016 . \quad$ doi: 10.1109/JLT.2016.2608138.

[11] E. P. Martin et al., "25-Gb/s OFDM 60-GHz Radio Over Fiber System Based on a Gain Switched Laser," in Journal of Lightwave Technology, vol. 33, no. 8, pp. 1635-1643, April15, 15 2015. doi: 10.1109/JLT.2015.2391994.

[12] C. H. Ho et al., "Performance Evaluation of a $60 \mathrm{GHz}$ Radio-over-Fiber System Employing MIMO and OFDM Modulation," in IEEE Journal on Selected Areas in Communications, vol. 31, no. 12, pp. 780-787, December 2013. doi: 10.1109/JSAC.2013.SUP2.12130010.

[13] H. Shams and J. Zhao, "First Investigation of fast OFDM Radio over fibre system at $60 \mathrm{GHz}$ using direct laser modulation," 2013 Conference on Lasers \& Electro-Optics Europe \& International Quantum Electronics Conference CLEO EUROPE/IQEC, Munich, 2013, pp. 1-1. doi: 10.1109/CLEOE-IQEC.2013.6801274.

[14] M. Chen, X. Xiao, J. Yu, X. Li and F. Li, "Real-Time Generation and Reception of OFDM Signals for X -Band RoF Uplink With Heterodyne Detection," in IEEE Photonics Technology Letters, vol. 29, no. 1, pp. 5154, Jan.1, 1 2017. doi: 10.1109/LPT.2016.2628758.

[15] S. H. Fan, J. Yu, D. Qian and G. K. Chang, "A Fast and Efficient Frequency Offset Correction Technique for Coherent Optical Orthogonal Frequency Division Multiplexing," in Journal of Lightwave Technology, vol. 29, no. 13, pp. 1997-2004, July 1, 2011. doi: 10.1109/JLT.2011.2156383

[16] P. H. Moose, "A technique for orthogonal frequency division multiplexing frequency offset correction," in IEEE Transactions on Communications, vol. 42, no. 10, pp. 2908-2914, Oct 1994. doi: $10.1109 / 26.328961$.

[17] T. M. Schmidl and D. C. Cox, "Robust frequency and timing synchronization for OFDM," in IEEE Transactions on Communications, vol. 45, no. 12, pp. 1613-1621, Dec 1997. doi: 10.1109/26.650240.

[18] Hlaing Minn, V. K. Bhargava and K. B. Letaief, "A robust timing and frequency synchronization for OFDM systems," in IEEE Transactions on Wireless Communications, vol. 2, no. 4, pp. 822-839, July 2003. doi: 10.1109/TWC.2003.814346.

[19]. Byungjoon Park, Hyunsoo Cheon, Changeon Kang and Daesik Hong, "A simple preamble for OFDM timing offset estimation," Proceedings IEEE 56th Vehicular Technology Conference, 2002, pp. 729-732 vol.2. doi: 10.1109/VETECF.2002.1040695.

[20] Guangliang Ren, Yilin Chang, Hui Zhang and Huining Zhang, "Synchronization method based on a new constant envelop preamble for OFDM systems," in IEEE Transactions on Broadcasting, vol. 51, no. 1, pp. 139-143, March 2005. doi: 10.1109/TBC.2004.842520.

[21] J. M. B. Oliveira, H. M. Salgado and M. R. D. Rodrigues, "A New MSE Channel Estimator Optimized for Nonlinearly Distorted Faded OFDM Signals With Applications to Radio Over Fiber," in IEEE Transactions on Communications, vol. 62, no. 8, pp. 2977-2985, Aug. 2014. doi:10.1109/TCOMM.2014.2334619. 